\title{
Pexidartinib in the Management of Advanced
}

\section{Tenosynovial Giant Cell Tumor: Focus on Patient Selection and Special Considerations}

\author{
Anna Vaynrub $\mathbb{D}^{\prime}$ \\ John $\mathrm{H}$ Healey $\left(\mathbb{D}^{2}\right.$ \\ William Tap $\mathbb{D}^{3}$ \\ Max Vaynrub $\mathbb{D}^{2}$ \\ 'Columbia University Vagelos College of \\ Physicians and Surgeons, New York, NY, \\ USA; ${ }^{2}$ Department of Surgery, Memorial \\ Sloan Kettering Cancer Center, New \\ York, NY, USA; ${ }^{3}$ Department of \\ Medicine, Memorial Sloan Kettering \\ Cancer Center, New York, NY, USA
}

Correspondence: Max Vaynrub

Department of Surgery, Memorial Sloan Kettering Cancer Center, 1275 York

Avenue, H-I0II, New York, NY, I0065, USA

Tel +I 2126397038

$\mathrm{Fax}+12127173573$

Email Max.Vaynrub@gmail.com

\begin{abstract}
Tenosynovial giant cell tumor (TGCT) is a neoplasm of the joint synovium that can have severe impacts on joint mobility, function, and quality of life. Traditionally, treatment modalities included partial or complete surgical synovectomy, radiotherapy (typically as an adjunct to surgery), and watchful monitoring (no medical or surgical intervention). However, these approaches have been met with varying degrees of success and high recurrence rates, as well as onerous complications and clinical sequelae. Pexidartinib, a colony-stimulating factor 1 receptor (CSF1R) inhibitor, presents a promising molecular approach that targets a neoplastic driver of TGCT. While the introduction of pexidartinib allows clinicians to avoid the significant morbidity associated with traditional treatment options, there are also defined risks associated with pexidartinib treatment. Therefore, patient selection is critical in optimizing treatment modalities in TGCT. The purpose of this literature review is to identify the TGCT patient population that would derive maximal benefit with minimal risk from pexidartinib, and to determine the specific indications and contraindications for selecting pexidartinib over other therapeutic approaches. Specifically, this paper compares the efficacy and safety profile of pexidartinib across clinical and preclinical studies to that of surgery, radiotherapy, and watchful monitoring. Rates of improvement in joint mobility, pain, and recurrence-free survival across studies of pexidartinib have been encouraging. The most common adverse events are mild (hypopigmentation of the hair) or reversible (transient aminotransferase elevation). Severe or permanent adverse events (notably cholestatic hepatotoxicity) are rare. While the optimal treatment strategy remains highly dependent on a patient's clinical circumstances and treatment goals, pexidartinib has surfaced as a promising therapeutic in cases where the morbidity of surgery or radiotherapy outweighs the benefits.
\end{abstract}

Keywords: pigmented villonodular synovitis, PVNS, giant cell tumor of tendon sheath, GCT-TS, colony-stimulating factor 1, CSF1, hepatotoxicity, radiotherapy, synovectomy

\section{Introduction}

Tenosynovial giant cell tumor (TGCT) occurs at a rate of 11-43 cases per million person-years and often presents with a complicated, unpredictable clinical course. ${ }^{1-3}$ The tumor originates in the synovium of joints, bursae, and tendon sheaths, and is considered a benign neoplastic process that results in a secondary inflammatory joint response. ${ }^{4}$ Characteristic symptomatology and pathophysiologic changes include pain and stiffness in the affected joint, swelling, hemorrhagic joint effusions, periarticular erosions, cartilage degradation, and secondary osteoarthritis; however, presentation varies widely depending on disease 
progression and anatomic site. ${ }^{1,2,4}$ While TGCT is usually not life-threatening, advanced disease can have a severe impact on quality of life. ${ }^{5}$

The clinical approach to TGCT can, in large part, be considered in terms of its classification as local or diffuse. In 2013, the World Health Organization reclassified diffuse pigmented villonodular synovitis (PVNS) as "diffuse-type giant cell tumor" and what was formerly known as giant cell tumor of tendon sheath (GCT-TS) as localized TGCT. ${ }^{6}$ Put concretely, localized TGCT presents as a single nodule, while diffuse TGCT manifests as multiple nodules throughout the synovial layer. ${ }^{2}$ Diffuse TGCT typically impacts larger joints - most commonly the knee, hip, and ankle - and constitutes $10-20 \%$ of TGCT cases. ${ }^{2,7,8}$ Significantly more common is localized TGCT, which accounts for $80-90 \%$ of all TGCT cases and is most frequently observed in the tendon sheaths of radial three digits. ${ }^{1,8}$ For TGCT of the hand, patients will commonly observe an initially painless mass that eventually grows and painfully impinges on surrounding anatomy. ${ }^{1}$ TGCT of the knee often presents as intermittent, spontaneous swelling that gradually progresses and becomes more painful. ${ }^{1}$ Most TGCTs are diagnosed in individuals between the ages of 20 and 50, and they occur at roughly the same frequency in men and women. ${ }^{2}$ However, diffuse TGCT tends to appear more commonly among younger patients and females. ${ }^{4}$ It is important to note that TGCT can present, albeit rarely, in malignant form, with risk for metastasis to lymph nodes and the lungs. ${ }^{1}$

TGCT has been found to overexpress colony-stimulating factor 1 (CSF1), most commonly as a result of the chromosomal translocation $\mathrm{t}(1 ; 2)\left(\mathrm{CSF} 1\right.$; COL6A3). ${ }^{9}$ Loci at 5q22-31, 11q11-12, and 8q21-22 have also been confirmed to act, albeit less frequently, as CSF1 translocation partners, and more translocation partners continue to be identified. ${ }^{10}$ However, the tumor consists chiefly of nonneoplastic macrophages that express the CSF1 receptor (CSF1R); only $2-16 \%$ of tumor cells overexpress CSF1, suggesting that the immune infiltrate recruited by CSF1 makes up most of the tumor mass. ${ }^{1}$ These immune cells, along with giant cells and osteoclasts, are recruited to the tumor by neoplastic cells that overexpress CSF1. Subsequent interaction of CSF1 and CSF1R promotes the phenotypic shift from monocyte to macrophage, leading to further tumor growth and inflammation. ${ }^{4,11}$ The role of CSF1 as the neoplastic driver makes inhibition of the CSF1 pathway an opportune target for pharmacological control of this disease.
Pexidartinib is an orally administered small-molecule tyrosine kinase inhibitor that selectively inhibits CSF1R along with c-kit receptor tyrosine kinase (KIT) and fmslike tyrosine kinase 3 internal tandem duplication (FLT3ITD). ${ }^{8}$ Various studies have validated pexidartinib as an efficacious systemic therapy for advanced TGCT in cases when surgery is considered excessively morbid or clinically unfeasible. Pexidartinib can also be utilized to reduce initial tumor burden in order to facilitate surgery.

The treatment approach to TGCT is guided by whether the tumor is localized or diffuse, by the patient's treatment history, and by the patient's eligibility for various treatment modalities. Common treatment strategies described in this review include observation, surgical excision, radiotherapy, and systemic treatment with pexidartinib. Proper patient selection for treatment with pexidartinib is accomplished by comparing its published outcomes and known risks with those of the other relevant treatment modalities in the context of the individual patient's clinical scenario.

\section{Treatment Modalities: Efficacies, Indications, and Additional Considerations \\ Observation}

Observation may be elected for patients with less-thansevere symptoms whose disease is not rapidly progressive. The risk of monitoring without active intervention is irreversible destruction of articular and periarticular structures, with progressive pain and impairment, that may eventually necessitate joint replacement or, rarely, amputation. In the ENLIVEN trial, patients randomized to the placebo group - who did not receive treatment outside of monitoring - reported no improvement in mobility, pain, or tumor size over the duration of the study. ${ }^{12}$ Monitoring is typically indicated for patients whose symptomatology is less severe than that of patients to whom surgery or systemic treatment is offered, and who have had few prior treatments. These patterns were demonstrated in the TGCT Observational Platform Project (TOPP), a recent multinational, multicenter prospective observational study in which the "watchful monitoring" approach was assigned primarily to patients with diffuse TGCT who had undergone only surgery prior to enrollment. ${ }^{7}$ At baseline, the watchful monitoring cohort in TOPP had lower pain and interference scores, lower frequency of painkiller use, and higher quality of life than patients for whom surgical or 
systemic treatment was indicated. ${ }^{7}$ However, the percentage of severe cases (instances of extra- and intra-articular disease with magnetic resonance imaging [MRI]-confirmed involvement of multiple muscles or ligaments) ${ }^{13}$ in this group was similar to that in the other cohorts, suggesting that clinical grade of the disease does not necessarily correlate with level of impairment. Thus, disease severity may not be a sufficient indicator of optimal treatment modality on its own, but rather should be considered in the context of the patient's symptomatology at presentation.

\section{Surgery}

Surgical excision of TGCT can be curative. However, surgical intervention can be invasive and morbid for the patient and, especially in diffuse TGCT, does not eliminate the risk of recurrence. For localized TGCT, the ideal treatment is complete resection of the tumor nodule with preservation of normal structures. In cases of diffuse TGCT in which the tumor distribution is such that the mechanics of and recovery from its removal will not compromise the patient's mobility more than the disease course itself, surgery is also the preferred treatment. This is especially true in cases affecting the hip, given the substantial load and rotational forces acting on the joint. ${ }^{14}$ Complete synovectomy is generally the first-choice method, although it often results in significant postoperative scarring and joint stiffness, particularly after an open approach. Additional postoperative morbidity may include hemarthrosis, joint instability, neurologic injury, thromboembolic disease, and wound healing complications. ${ }^{2}$ An international multicenter review by Mastboom et al found that $12 \%$ of TGCT patients treated with surgery experienced surgical complications (Table 1). ${ }^{13}$ The choice of open versus arthroscopic synovectomy can be controversial, but some data suggest that open synovectomy leads to lower recurrence (14\%, compared with $40 \%$ following arthroscopic synovectomy). ${ }^{1,2,4}$

In their review, Mastboom et al found that $44 \%$ of surgically treated TGCT patients developed disease recurrence, and that the prevalence of recurrence-free survival was $62 \%$ at three years, $55 \%$ at five years, and $40 \%$ at 10 years. ${ }^{13}$ Fifty-nine percent of patients saw a reduction in pain and $72 \%$ had diminished swelling. While surgical resection has a high local control rate for localized cases, $44-72 \%$ of resected diffuse TGCT cases recur, ${ }^{2,8,13,15,16}$ underscoring the need for alternative or complementary therapies.
The limited long-term success of surgery for diffuse TGCT is further compounded by the technical challenge these cases often present. Diffuse TGCT frequently occurs concurrently with secondary osteoarthritis as a consequence of associated disease pathology or of the surgical management strategies themselves. ${ }^{2}$ Studies suggest that joint replacement combined with complete synovectomy is associated with lower recurrence rates than surgery alone, likely because the synovial membrane can be completely removed through the joint replacement procedure. $^{2}$ Nevertheless, the recurrence rate after joint replacement and synovectomy remains high, mitigating the delay of medical or surgical treatment on the assumption that resection and arthroplasty will ultimately control the diffuse TGCT. While total knee arthroplasty (in conjunction with total synovectomy) can offer pain-free joint function, prostheses implantation in the setting of diffuse TGCT has been linked with subsequent joint stiffness and high rates of revision. ${ }^{2}$ Thus, the presence of concurrent conditions that may compound the risk of surgery and complicate functional recovery should be weighed in the decision between surgical and systemic approaches.

\section{Radiotherapy}

Radiotherapies such as radiosynoviorthesis (RSO) and external beam radiotherapy (EBRT) have been employed as adjuncts to surgery. The potential benefits of radiotherapy are relapse prevention (via inhibition of cellular proliferation) and pain relief. ${ }^{17}$ Postoperative radiotherapy is associated with high rates of local control: Griffin et al observed a 94\% local control rate among patients with severe or recurrent TGCT; ${ }^{14}$ Berger et al reported a $100 \%$ local control rate in 7 patients at a mean follow-up of 29 months; ${ }^{18}$ Blanco et al attained a rate of $86 \%$ at a mean follow-up of 33 months; ${ }^{19}$ and Horoschak et al had an initial control rate of $75 \%$ at a mean of 48 months of follow-up. ${ }^{20}$

RSO most commonly involves implantation of colloid labeled with ${ }^{90}$ yttrium into the target joint. ${ }^{2,17}$ The applicability of this approach is limited by its invasive nature, by the financial burden it poses, and by the associated risk of early onset arthritis, avascular necrosis, and healing complications. Case reviews found that patients treated with RSO after surgery experienced a recurrence rate of $23-44 \%$, compared with the $44-72 \%$ rate observed in patients treated with surgery only. ${ }^{7,13,16,17,21,22}$ A retrospective single-center case review found that patients treated with RSO in addition to open synovectomy had a 
Table I Overview of Key Reviewed Studies

\begin{tabular}{|c|c|c|c|}
\hline Study & Type & Patient Population & Results \\
\hline $\begin{array}{l}\text { Giustini } N \\
\text { et al }\end{array}$ & Case report & $\begin{array}{l}\text { I TGCT patient who was not considered } \\
\text { for surgery due to excessive morbidity; } \\
\text { treated with pexidartinib for } 4 \text { months }\end{array}$ & $\begin{array}{l}\text { - Tumor volume on MRI had decreased by } 48 \% \text { at } 4 \\
\text { months according to RECIST criteria } \\
\text { - Disease was stable at } 55 \text { months }\end{array}$ \\
\hline $\begin{array}{l}\text { Gelderblom } \\
\mathrm{H} \text { et al }\end{array}$ & $\begin{array}{l}\text { Retrospective pooled analysis of } 3 \\
\text { pexidartinib studies: } \\
\text { I. Phase I extension study }(1000 \mathrm{mg} / \\
\text { d) } \\
\text { 2. ENLIVEN patients given } \\
\text { pexidartinib ( } 1000 \mathrm{mg} / \mathrm{d} \text { for } 2 \text { weeks } \\
\text { and } 800 \mathrm{mg} / \mathrm{d} \text { for } 22 \text { weeks) } \\
\text { 3. ENLIVEN crossover patients ( } 800 \\
\mathrm{mg} / \mathrm{d} \text { ) }\end{array}$ & $\begin{array}{l}\text { I } 30 \text { patients with symptomatic, } \\
\text { unresectable TGCT confirmed on histology } \\
(39,61 \text {, and } 30 \text { patients })\end{array}$ & $\begin{array}{l}\text { By RECIST criteria: } \\
\text { - Overall response rate: } 60 \% \\
\text { - Median time to response: } 3.4 \text { months } \\
\text { - Among responders, } 62 \% \text { had partial response by } 6 \\
\text { months and } 92 \% \text { by } 18 \text { months } \\
\text { By tumor volume score: } \\
\text { - Overall response rate: } 65 \% \\
\text { - Median time to response: } 2.8 \text { months } \\
\text { - Median duration of response: } 47 \text { months } \\
\text { Treatment-emergent adverse events } \\
\text { - Hair color changes: } 75 \% \\
\text { - Aminotransferase elevations: } 92 \% \\
\text { - Mixed/cholestatic hepatotoxicity: } 3 \% \text { (all cases } \\
\text { were in first } 2 \text { months of treatment and were } \\
\text { reversible) }\end{array}$ \\
\hline $\begin{array}{l}\text { Tap WD } \\
\text { et } \mathrm{al}^{12}\end{array}$ & $\begin{array}{l}\text { Phase } 3 \text { randomized trial. } \\
\text { - Part I: Double-blind study of } \\
\text { patients assigned to pexidartinib } \\
\text { ( } 000 \mathrm{mg} / \mathrm{d} \text { for } 2 \text { weeks, then } 800 \\
\mathrm{mg} / \mathrm{d} \text { for } 22 \text { weeks) or placebo. } \\
\text { - Part 2: Open-label treatment with } \\
\text { pexidartinib for all patients. }\end{array}$ & $\begin{array}{l}\text { I } 20 \text { patients with symptomatic, advanced } \\
\text { TGCT for whom surgery was not } \\
\text { recommended }\end{array}$ & $\begin{array}{l}\text { Overall response by RECIST at } 25 \text { weeks } \\
\text { - Pexidartinib: } 39 \% \\
\text { - Placebo: } 0 \% \\
\text { - Absolute difference: } 39 \% \text { ( } 95 \% \mathrm{Cl}, 27-53 \% \text {; } \\
\text { p<0.000I) } \\
\text { Serious adverse events } \\
\text { - Pexidartinib: I } 3 \% \\
\text { - Placebo: } 2 \% \\
\text { - Three pexidartinib recipients had liver enzyme } \\
\text { elevations suggesting cholestatic hepatotoxicity, } \\
\text { including one case confirmed by biopsy } \\
\text { Pexidartinib-associated adverse events: } \\
\text { - Hair color changes ( } 67 \%), \text { fatigue ( } 54 \%) \text {, aspartate } \\
\text { aminotransferase increase ( } 39 \%), \text { nausea ( } 38 \%) \text {, } \\
\text { alanine aminotransferase increase ( } 28 \%), \text { dysgeusia } \\
(25 \%)\end{array}$ \\
\hline $\begin{array}{l}\text { Mastboom } \\
\text { MJL et } \text { al }^{13}\end{array}$ & $\begin{array}{l}\text { International multicenter } \\
\text { retrospective cohort study }\end{array}$ & $\begin{array}{l}966 \text { patients with histologically proven } \\
\text { diffuse-type TGCT of large joint who were } \\
\text { treated with surgery in } 31 \text { sarcoma } \\
\text { reference centers between } 1990 \text { and } 2017\end{array}$ & $\begin{array}{l}\text { - Tumor recurred in } 44 \% \text { of patients by follow up } \\
\text { (median, } 54 \text { months; IQR, } 27-97 \text { months) } \\
\text { - Prevalence of recurrence-free survival was } 62 \% \text { at } \\
3 \text { years, } 55 \% \text { at } 5 \text { years, and } 40 \% \text { at } 10 \text { years } \\
\text { - } 12 \% \text { of patients with complete data had surgical } \\
\text { complications }\end{array}$ \\
\hline $\begin{array}{l}\text { Griffin AM } \\
\text { et } \mathrm{al}^{14}\end{array}$ & Retrospective review & $\begin{array}{l}50 \text { patients treated with radiotherapy and } \\
\text { surgery between } 1972 \text { and } 2006.49 \text { had } \\
\text { diffuse TGCT with both intra-articular and } \\
\text { extra-articular disease; I had malignant } \\
\text { TGCT }\end{array}$ & $\begin{array}{l}\text { - } 30 \text { patients }(60 \%) \text { underwent at least } 2 \text { operations } \\
\text { before radiotherapy } \\
\text { At follow up (mean, } 94 \text { months): } \\
\text { - } 47 \text { patients }(94 \%) \text { had stable disease or no } \\
\text { recurrence on serial cross-sectional imaging } \\
\text { - } 2 \text { patients had had total hip arthroplasty due to } \\
\text { progressive osteoarthritis } \\
\text { - } 4 \text { patients had had avascular necrosis, one of } \\
\text { which occurred posttreatment }\end{array}$ \\
\hline
\end{tabular}

(Continued) 
Table I (Continued).

\begin{tabular}{|c|c|c|c|}
\hline Study & Type & Patient Population & Results \\
\hline $\begin{array}{l}\text { Mollon B } \\
\text { et } \text { al }^{15}\end{array}$ & Systematic review/meta-analysis & $\begin{array}{l}630 \text { patients in } 35 \text { observational studies of } \\
\text { surgical synovectomy to treat PVNS of the } \\
\text { knee }\end{array}$ & $\begin{array}{l}\text { - I } 37 \text { patients }(22 \%) \text { had recurrence after } \\
\text { synovectomy } \\
\text { - Analysis found "low-quality" evidence that } \\
\text { recurrence risk was lower for open synovectomy } \\
(\mathrm{OR}, 0.47 ; 95 \% \mathrm{Cl}, 0.25-0.90) \text { and for combined } \\
\text { open and arthroscopic synovectomy (OR, } 0.19 ; 95 \% \\
\mathrm{Cl}, 0.06-0.58) \text { than for arthroscopic surgery } \\
\text { - Analysis found "very low-quality evidence" that } \\
\text { perioperative radiotherapy reduces recurrence risk } \\
(\mathrm{OR}, 0.3 \mathrm{I} ; 95 \% \mathrm{Cl}, 0.14-0.70)\end{array}$ \\
\hline $\begin{array}{l}\text { Xiang } X \\
\text { et al }{ }^{17}\end{array}$ & Case study report & $\begin{array}{l}\text { Two males with knee swelling who } \\
\text { underwent image-guided, intensity- } \\
\text { modulated radiotherapy after incomplete } \\
\text { synovectomy. Diffuse TGCT was confirmed } \\
\text { by postoperative pathology. }\end{array}$ & $\begin{array}{l}\text { - At one year, no disease progression was evident } \\
\text { on MRI } \\
\text { - No adverse events were attributed to } \\
\text { radiotherapy during follow up }\end{array}$ \\
\hline $\begin{array}{l}\text { Berger B } \\
\text { et } \mathrm{al}^{18}\end{array}$ & Retrospective review & $\begin{array}{l}7 \text { patients with diffuse PVNS, mostly at the } \\
\text { knee, who were treated with } 6 \text {-millivolt } \\
\text { photo radiotherapy after radical surgery }\end{array}$ & $\begin{array}{l}\text { - No persistent or recurrent disease or acute or } \\
\text { late adverse effects were observed at follow-up } \\
\text { (mean, } 29 \text { months; range, 3-112 months) } \\
\text { - } 6 \text { patients reported excellent quality of life and } \\
\text { "asymptomatic limb function" } \\
\text { - I patient reported persistent restriction of joint } \\
\text { movement following multiple surgeries }\end{array}$ \\
\hline 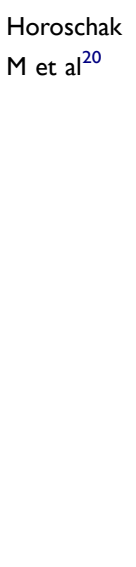 & Retrospective review & $\begin{array}{l}17 \text { PVNS patients with } 18 \text { sites of disease } \\
\text { who were treated with radiation between } \\
1993 \text { and } 2007\end{array}$ & $\begin{array}{l}\text { - During follow-up (mean, } 46 \text { months), } 12 \text { of } 16 \\
\text { sites }(75 \%) \text { with previous cytoreductive surgery } \\
\text { displayed initial local control (mean time to } \\
\text { recurrence, } 38 \text { months) } \\
\text { - Ultimate local control after repeat resection was } \\
100 \% \text { (mean follow-up, } 61 \text { months) } \\
\text { - Growth occurred in } 2 \text { sites without prior } \\
\text { cytoreductive surgery after radiotherapy (mean } \\
\text { time to documented growth, } 10.5 \text { months) } \\
\text { - I7/I8 involved joints ( } 94 \% \text { ) had excellent or good } \\
\text { PVNS-related function; one site (6\%) had fair } \\
\text { function } \\
\text { - No patients underwent amputation or had grade } \\
3 / 4 \text { adverse events }\end{array}$ \\
\hline $\begin{array}{l}\text { Dürr HR } \\
\text { et } \mathrm{al}^{22}\end{array}$ & Retrospective review & $\begin{array}{l}32 \text { patients with diffuse PVNS who } \\
\text { underwent a total of } 37 \text { open } \\
\text { synovectomies from } 1996 \text { to } 2014 \text {; adjuvant } \\
\text { radiosynoviorthesis was administered } \\
\text { following } 26 \text { of the resections }\end{array}$ & $\begin{array}{l}\text { - } 9 \text { lesions }(24 \%) \text { had recurred by follow-up } \\
\text { (median, } 19 \text { months postsurgery)-3 in cases of } \\
\text { primary disease and } 6 \text { in already recurring cases } \\
\text { - Recurrence occurred in } 6 / 26(23 \%) \text { cases in which } \\
\text { radiosynoviorthesis was performed and } 3 / 1 \text { I (27\%) } \\
\text { cases without radiosynoviorthesis }\end{array}$ \\
\hline $\begin{array}{l}\text { Ottaviani S } \\
\text { et } \mathrm{al}^{23}\end{array}$ & Retrospective review & $\begin{array}{l}\text { I22 patients with PVNS confirmed on } \\
\text { histology; } 98 \% \text { underwent synovectomy }\end{array}$ & $\begin{array}{l}- \text { During follow up (mean, } 5.8 \text { years), the relapse } \\
\text { rate was } 30 \% \text { (knee) and } 9 \% \text { (other sites) for } \\
\text { patients with diffuse disease treated with surgery } \\
\text { and then isotopic synoviorthesis } \\
\text { - Mean time to relapse was } 2.6 \text { years (knee) and } 2.4 \\
\text { years (other sites) }\end{array}$ \\
\hline
\end{tabular}

(Continued) 
Table I (Continued).

\begin{tabular}{|c|c|c|c|}
\hline Study & Type & Patient Population & Results \\
\hline $\begin{array}{l}\text { Park G } \\
\text { et } \mathrm{al}^{24}\end{array}$ & Retrospective study & $\begin{array}{l}23 \text { patients who received radiotherapy after } \\
\text { synovectomy between } 1998 \text { and } 2007\end{array}$ & $\begin{array}{l}\text { - During follow-up (median, } 9 \text { years; range, } 0.8-12 \\
\text { years), } 4 \text { patients had disease recurrence (median } \\
\text { time to recurrence, } 5 \text { years); local control was } \\
\text { achieved for three of them though salvage } \\
\text { synovectomy } \\
\text { - } 22 \text { patients reported excellent or good joint } \\
\text { function, while I who did not receive salvage } \\
\text { synovectomy reported poor joint function } \\
\text { - No grade } 3 \text { toxicities or radiation-related } \\
\text { malignancies were documented }\end{array}$ \\
\hline $\begin{array}{l}\text { Lewis JH } \\
\text { et } \mathrm{al}^{32}\end{array}$ & $\begin{array}{l}\text { Pooled analysis of safety data from } 4 \\
\text { clinical trials of pexidartinib }\end{array}$ & $\begin{array}{l}\text { I } 40 \text { TGCT patients and } 658 \text { patients with } \\
\text { various malignancies }\end{array}$ & $\begin{array}{l}\text { - Median follow up: } 39 \text { months } \\
\text { - Hepatic adverse reactions were seen in } 5 \% \text { of } \\
\text { TGCT patients (median duration of exposure, } 19 \\
\text { months) } \\
\text { - } 128 \text { TGCT patients ( } 91 \% \text { ) had reversible dose- } \\
\text { dependent AST/ALT elevations without ALP } \\
\text { elevation } \\
\text { - } 5 \text { TGCT patients (4\%) experienced mixed or } \\
\text { cholestatic hepatotoxicity; all recovered within } 7 \\
\text { months of pexidartinib discontinuation } \\
\text { - } 5 \text { non-TGCT patients ( } 1 \% \text { ) had severe hepatic } \\
\text { adverse reactions, two of which were irreversible }\end{array}$ \\
\hline $\begin{array}{l}\text { Tap WD } \\
\text { et } \mathrm{al}^{33}\end{array}$ & $\begin{array}{l}\text { Multicenter phase I trial: } \\
\text { - Part I: Dose-escalation study of } \\
\text { pexidartinib administered orally to } \\
\text { patients with solid tumors. } \\
\text { - Part 2: Extension study } \\
\text { investigating pexidartinib }(100 \mathrm{mg} / \mathrm{d}) \\
\text { in TGCT patients. }\end{array}$ & $\begin{array}{l}\text { - Part I: } 4 \text { I solid tumor patients } \\
\text { - Part 2: } 23 \text { TGCT patients }\end{array}$ & $\begin{array}{l}\text { - Median duration of response: }>8 \text { months } \\
\text { - Responses generally seen within } 4 \text { months of } \\
\text { treatment initiation } \\
\text { - Most frequent adverse events: fatigue, change in } \\
\text { hair color, nausea, dysgeusia, periorbital edema } \\
\text { - Decrease in tumor volume was sustained in most } \\
\text { patients who responded to treatment }\end{array}$ \\
\hline $\begin{array}{l}\text { Van De } \\
\text { Sande M } \\
\text { et al }{ }^{34}\end{array}$ & $\begin{array}{l}\text { Analysis of patient-reported } \\
\text { outcome data from ENLIVEN } \\
\text { (double-blinded, randomized phase } 3 \\
\text { trial) }\end{array}$ & Adults with symptomatic, advanced TGCT & $\begin{array}{l}\text { Change in physical function at } 25 \text { weeks (assessed } \\
\text { using Patient-Reported Outcomes Measurement } \\
\text { Information System-Physical Function) } \\
\text { - Pexidartinib: } 4.1(95 \% \mathrm{Cl}, \mathrm{I} .8-6.3) \\
\text { - Placebo: }-0.9(95 \% \mathrm{Cl},-3.0 \text { to I.2) } \\
\text { Change in worst stiffness (assessed using a } \\
\text { numerical rating scale) } \\
\text { - Pexidartinib: }-2.5(95 \% \mathrm{Cl},-3.0 \text { to }-1.9) \\
\text { - Placebo: }-0.3 \text { ( } 95 \% \mathrm{Cl},-0.9 \text { to } 0.3) \\
\text { - Improvements were maintained after } 50 \text { weeks of } \\
\text { pexidartinib treatment }\end{array}$ \\
\hline $\begin{array}{l}\text { Smith CC } \\
\text { et } \mathrm{al}^{38}\end{array}$ & $\begin{array}{l}\text { Phase I/2 study } \\
\text { - Part I: Dose escalation } \\
\text { - Part 2: Dose expansion }\end{array}$ & $\begin{array}{l}90 \text { patients ( } 34 \text { part I, } 56 \text { part } 2 \text { ) with } \\
\text { relapsed or refractory FLT3-ITD-mutant } \\
\text { acute myeloid leukemia }\end{array}$ & $\begin{array}{l}\text { - Most common adverse events were diarrhea } \\
(50 \%) \text {, fatigue }(47 \%) \text {, nausea }(46 \%) \\
\text { - Overall response rate: } 21 \% \\
\text { - Median overall survival in dose expansion cohort: } \\
\text { II } 2 \text { days ( } 90 \% \mathrm{Cl}, 77-150 \text { days) } \\
\text { - Median overall survival of those in complete } \\
\text { remission: } 265 \text { days ( } 90 \% \mathrm{Cl}, 170-422 \text { days) }\end{array}$ \\
\hline
\end{tabular}

Abbreviations: TGCT, tenosynovial giant cell tumor; MRI, magnetic resonance imaging; RECIST, response evaluation criteria in solid tumors; $\mathrm{Cl}$, confidence interval; IQR, interquartile range; PVNS, pigmented villonodular synovitis; OR, odds ratio; AST, aspartate aminotransferase; ALT, alanine aminotransferase; ALP, alkaline phosphatase. 
recurrence rate of $30 \%$ for tumors of the knee (and $9 \%$ for tumors located elsewhere) after a mean of 4.6 years of follow-up. ${ }^{23}$ Another retrospective review found that administering RSO did not lead to improvements in physical and mental health, pain, or satisfaction at a mean follow up of 7.3 years. $^{21}$

EBRT is less invasive than RSO and involves only a computed tomography planning system and linear accelerator. ${ }^{17}$ Studies have found recurrence rates of up to $17 \%$ among diffuse TGCT patients receiving EBRT as a surgical adjuvant at a median follow-up of 9 years. $^{24}$ Downstream sequelae and complications of EBRT include dermatological reactions, impaired wound healing, stiffness, and malignant transformation. ${ }^{2}$ The nature of these risks suggests that EBRT should be reserved for cases of symptomatic and recurrent diffuse TGCT for which other treatments are contraindicated or ineffectual and that, whenever possible, nonradiation modalities should be prioritized.

Because radiotherapy is typically employed as an adjunct to surgery, minimal data exist regarding its utility as a monotherapy. In a retrospective analysis evaluating patients with diffuse TGCT, no patients who received definitive radiotherapy (radiotherapy alone without surgery) experienced recurrence. ${ }^{25}$ TGCT recurred in $15 \%$ of the total cohort, and all of these individuals underwent radiotherapy postoperatively. Interestingly, obesity was identified as a predictive factor for local recurrence. Complications from radiotherapy included grade 1 dermatitis, joint effusion, and limited range of motion. In a retrospective review led by de Visser, 2 of 38 TGCT patients received radiosynovectomy with ${ }^{90}$ yttrium as sole treatment for tumor of the knee. ${ }^{26}$ One of these patients had residual tumor following treatment and the other experienced disease recurrence. Both patients had a functional outcome rating of "fair." In 2009, Horoschak et al observed that both patients in their cohort who had not undergone cytoreductive surgery prior to radiotherapy saw disease recurrence (after an average time of 10.5 months). In contrast, $100 \%$ of patients who had undergone surgery before radiotherapy had ultimate local control at a mean follow-up of 61 months, though $25 \%$ had a recurrence between initial surgery and this timepoint. ${ }^{20}$

\section{Pexidartinib}

Historically, the clinical success of relatively strong CSF1 inhibitors for the treatment of TGCT has been disappointing. In a 2015 Phase 1 trial, treatment with the CSF1R monoclonal antibody emactuzumab achieved a $7 \%$ complete response rate and a $79 \%$ partial response rate at 12 months' follow up in a cohort patients with advanced diffuse TGCT; most adverse events (all of which had an event rate of over $50 \%$ ) were grade 1 or 2 in severity. ${ }^{27}$ Since this study, however, minimal data on emactuzumab has been released. ${ }^{28}$ In a Phase 2 trial of nilotinib, a CSF1R tyrosine kinase inhibitor, over $90 \%$ of treated patients had stable disease at 12 weeks, but the objective treatment response was $0 \%$ at that time point, and only $6 \%$ during the one-year study period. ${ }^{29}$ Ninety-six percent of patients experienced treatment-related adverse events. Imatinib, a tyrosine kinase inhibitor with activity against CSF1R, produced an overall response rate of $19 \%$ with relatively few severe adverse events (though $21 \%$ of patients withdrew from the study due to treatment toxicity). ${ }^{30}$ DCC3014 (vimseltinib) has shown positive biomarker modulation in TGCT patients and is currently being evaluated in phase 1 and 2 trials. $^{31}$

Encouragingly, the efficacy of pexidartinib has been demonstrated across several studies. Recently, the National Comprehensive Cancer Network designated pexidartinib a category 1 recommendation for adult patients who have symptomatic TGCT and severe morbidity and for whom surgery is not an option. ${ }^{32}$

The first human study to find evidence of the drug's effectiveness was PLX108-01, a phase 1 extension study with dose escalation. ${ }^{33}$ Positive results from that study led to the ENLIVEN trial, a landmark multicenter doubleblinded, placebo-controlled Phase 3 study of pexidartinib in patients with TGCT. ${ }^{12}$ Tumor response was assessed using Response Evaluation Criteria in Solid Tumors (RECIST) as a primary endpoint and tumor volume score (TVS) as a secondary endpoint. Patients receiving pexidartinib had an overall response rate of 39\% (as measured by RECIST) at week 25 , compared with $0 \%$ in the control group ( $p<0.0001)$. In the analysis of TVS, the pexidartinib group had an overall response rate of $56 \%$, compared with $0 \%$ in the control group $(\mathrm{p}<0.0001)$. Overall response rates were even greater $-56 \%$ by RECIST and $64 \%$ by TVSat the study's data cutoff timepoint (median follow-up, 22 months). Furthermore, the responses proved durable, with few instances of progression in responders. Long-term outcome was assessed in a follow-up study that extended 26 months after the ENLIVEN data cutoff; overall response rates among pexidartinib-treated patients were $60 \%$ by RECIST and $65 \%$ by TVS. $^{8}$ In a pooled analysis of PLX108-01 and ENLIVEN data, median duration of 
response by TVS was 46.8 months and was not reached by RECIST.

Moreover, pexidartinib-treated patients demonstrated significantly better patient-reported outcomes than placebo recipients. ${ }^{34}$ Specifically, between baseline and week 25 , patients treated with pexidartinib had a mean improvement of 4.1 points in Patient-Reported Outcomes Measurements Information System-Physical Function (PROMIS-PF) scores (which reflect patient mobility), while placebo patients had a mean decline of 0.9 points. Pexidartinib patients also had improvement in Worst Stiffness Numeric Rating Scale scores (a 2.5-point reduction on average, compared to a 0.3 -point decline among placebo patients). Changes of 3 points in physical function scores and 1 point in stiffness scores can be considered clinically meaningful ${ }^{35}$ by these standards, pexidartinib produced clinically relevant improvement in functional outcomes, which aligns with the conclusion that tumor response correlated with functional improvement. ${ }^{34}$

In 2019, the US Food and Drug Administration (FDA) approved pexidartinib for use in patients with advanced disease for whom a surgical approach is not available or medically feasible. ${ }^{8,36}$ However, the European Medicines Agency's Committee for Medicinal Products for Human Use refused authorization for pexidartinib, citing the risk of hepatotoxicity and the committee's belief that the symptomatic benefits were of insufficient degree and unknown duration. ${ }^{37}$

Several population-specific studies of pexidartinib outcomes have been undertaken. An open-label phase 1/2 dose-escalation trial found pexidartinib to be safe, well tolerated, and efficacious in patients with relapsed/refractory FLT3-ITD-mutant acute myeloid leukemia. Pexidartinib plasma exposure levels plateaued at a dosage of $3000 \mathrm{mg} / \mathrm{day}$, and no maximum tolerated dose was reached. ${ }^{38}$ The Phase I open-label multiple-dose study NCT02734433 looked exclusively at Asian patients with advanced solid tumors. ${ }^{39}$ While just one patient in the study was diagnosed with TGCT, this patient was also the only one to have an objective partial tumor response (as determined by RECIST). Overall, the authors concluded that the safety and efficacy profile of pexidartinib among Asian patients is similar to that among Western patients.

In defining optimal pexidartinib candidacy and indications, it is important to characterize the patient cohorts in which the drug has been studied. Most of the cohorts in published trials had a mean age in the 40 s and were predominantly female. ${ }^{7}$ Patients in the ENLIVEN study all had pain and stiffness scores of at least 4 on the relevant numeric rating scales. ${ }^{12}$ Of note, the majority of the ENLIVEN patients had TGCT of the knee and ankle. Another important characteristic of this population is that $53 \%$ of the pexidartinib cohort had undergone at least one previous surgery for TGCT. In addition, $85 \%$ of patients in the cohort were white and $57 \%$ were women; they had a median age of 44 (range, 22-75), a mean TVS of 14.8 (standard deviation, 21.2), and a mean tumor diameter of $101 \mathrm{~mm}$ (standard deviation, $63 \mathrm{~mm}$ ). Unfortunately, analyses of efficacy endpoints were not stratified by baseline data, limiting our understanding of potential differential effects across baseline demographic and tumor characteristics.

The primary safety concerns observed were hepatotoxic effects, thought to be due to pexidartinib's impact on Kupffer cells. ${ }^{12}$ Specifically, pexidartinib has been associated with two distinct classes of hepatic adverse reactions: reversible aminotransferase (alanine [ALT] or aspartate aminotransferase [AST]) elevations, and an idiopathic mixed or cholestatic hepatotoxicity. ${ }^{32}$ Aminotransferase elevations were relatively frequent, dose-dependent, and generally low grade, and occurred in the absence of bilirubin and alkaline phosphatase (ALP) elevations. ${ }^{8,32}$ Mixed or cholestatic hepatotoxicity, in contrast, featured an increase in ALP with or without aminotransferase elevation. ${ }^{32}$ These cases were less frequent, longer in duration, and more severe in nature, posing risk of irreversible hepatic damage. ${ }^{8,32}$ In a retrospective pooled review by Lewis et al, $80 \%$ of patients who developed cholestatic hepatotoxicity were female, a figure considerably higher than the proportion of trial enrollees who were women (57\%), though there was no statistical significance attached to this finding. ${ }^{32}$ Liver enzyme elevations and adverse events were also more severe in women: compared with men, women were more likely to have elevations of grade 3 or higher in AST $(17 \%$ vs $3 \%)$, ALT ( $20 \%$ vs $10 \%)$, and ALP (6\% vs $0 \%) .^{32}$ In the ENLIVEN study, $39 \%$ of patients in the pexidartinib cohort experienced AST elevations (10\% were grade $\geq 3)$ and $28 \%$ had ALT elevations ( $10 \%$ were grade $\geq 3) .{ }^{12}$ Severe hepatotoxic effects have also been disproportionately common among female patients in other studies. ${ }^{32}$ The risk of hepatotoxicity prompted the FDA to mandate monitoring of patients on pexidartinib under the Risk Evaluation and Mitigation Strategies (REMS). ${ }^{40}$ Thus, considering the hepatic health of patients 
is critical in determining pexidartinib candidacy and monitoring. Patients who are suffering from liver failure or injury, have comorbidities that impact liver function, or are taking drugs with hepatic risks may not be candidates for pexidartinib, and certainly warrant close monitoring (to a degree at least as stringent as the REMS) if they do initiate treatment.

Pexidartinib undergoes oxidation by CYP3A4 enzymes and glucuronidation by UGT1A4. ${ }^{41}$ Patients taking CYP3A or UGT inhibitors are therefore at increased risk of developing adverse reactions due to systemic accumulation of unmetabolized drug. Conversely, concurrent use of CYP3A inducers may limit pexidartinib's efficacy. Drugs such as proton pump inhibitors have also been shown to lower the area under the curve (AUC) of pexidartinib; however, pexidartinib may be administered two hours before or after a local antacid, and two hours prior or 10 hours after an $\mathrm{H}_{2}$-receptor antagonist. ${ }^{36,41}$ Therefore, patients for whom acid reduction therapies are essential should be carefully considered but not dismissed as potential beneficiaries.

Pexidartinib's pharmacokinetics do not differ significantly by patients' age, sex, or race (White or Black), or with mild hepatic impairment. However, pexidartinib exposure (AUC) is $30 \%$ greater in patients with renal impairment (as defined by creatinine clearance) than in those with normal renal function. Thus, dosage reduction is necessary in patients with renal impairment; the recommended regimen is $200 \mathrm{mg}$ in the morning and $400 \mathrm{mg}$ in the evening. ${ }^{36}$

Animal studies suggest a potential risk of infertility in both males and females, as well as a risk of fetal injury. ${ }^{41}$ In animal studies, rats and rabbits given pexidartinib at exposures equivalent to recommended human doses during organogenesis experienced elevated rates of postimplantation loss, fetal malformation, and abortion. ${ }^{36}$ Fetal deformities observed in rats given pexidartinib included such developmental abnormalities as localized fetal edema, absent kidney and ureter, abnormal renal formations, reproductive system defects, and decreased skeletal ossification. At exposures about 1.3 times the recommended human dosage, there was a decrease in pregnancy rate, a higher rate of fetal loss, and negative impacts on spermatogenesis and on sperm concentration, motility, and morphology. ${ }^{36}$ Decreases in testicular and epididymal weights and in hypospermia were seen in rats at only 0.3 and 0.6 times the exposure at the recommended human dose, respectively. Female rats experienced increased corpora lutea necrosis and hemorrhage and increased luteal cysts at only 0.01 times the human exposure at the recommended dose. Defects in reproductive structures were also seen in dogs at similarly low doses. Thus, reproductive-aged females and males should approach this treatment cautiously.

\section{Goals of Treatment: Defining Relative Morbidity}

The decision to manage a patient with treatment or observation directly relates not only to the severity of symptoms at presentation, but also to the projected disease course and the added difficulty of treatment at a more advanced stage of disease. Studies have found a moderate correlation between decrease in tumor size and improvement in PROMIS-PF scores and Worst Stiffness Numeric Rating Scale scores. ${ }^{34}$ These findings indicate that reducing tumor size and impeding progression can alter the natural history of functional deterioration; however, the correlation between tumor activity and function is imperfect, suggesting that irreversible alteration of articular structures may have occurred by the time tumor control is established. Therefore, the risks of treatment-related adverse events must be weighed against the risks of irreversible joint damage with observation.

Developing a baseline for acceptable morbidity requires understanding of natural disease course, recovery outcomes with surgery, and individual patient risks and limitations. One way to obtain this insight is to assess disease progression in individuals assigned to the placebo group in blinded trials. In the ENLIVEN study, $0 \%$ of patients in the placebo group achieved tumor response by RECIST and TVS by week $25 .{ }^{12}$ However, $78 \%$ of placebo patients had stable disease as measured by RECIST, and $2 \%$ experienced progressive disease. During the same 25-week period, functional outcomes, as measured by PROMIS-PF, trended worse $(-0.9 ; 95 \%$ confidence interval, -3.0 to 1.2$).^{34}$

The establishment of the TOPP registry represents an important opportunity for us to understand the natural history of TGCT, in the form of prospective observation of patients assigned to the watchful waiting group. However, even in this prospective study, the presence of selection bias (eg, referral of more severe cases to participating tertiary sarcoma centers, less severe disease in patients assigned to watchful waiting) prevents us from obtaining a completely accurate picture of the natural 
history of TGCT, though the resulting data likely provide the best available understanding of this rare disease's progression.

With baseline risks of disease progression in mind, we can compare the risks of pexidartinib therapy to those of observation or other available treatments. Notably, the rate of treatment-emergent adverse events of any grade in the placebo group was $93 \%$, comparable to the rate of $98 \%$ observed among pexidartinib patients. Among low-grade events, the most notable difference between groups was an increased risk of hypopigmentation of the hair in the pexidartinib group. ${ }^{12}$ However, compared with the placebo group, the pexidartinib cohort had a higher rate of grade 3 or 4 adverse events ( $44 \%$ vs $12 \%$ ), including AST, ALT, and ALP elevations and hypertension. ${ }^{12}$ One patient who received pexidartinib (outside of a TGCT trial) experienced fatal cholestatic hepatotoxicity. This individual was a 66-year-old female suffering from advanced mucosal melanoma who experienced drug-induced hepatitis with a total bilirubin level 3 times that of the upper limit of normal by day 25 of treatment. ${ }^{12,32}$ Current data from retrospective studies and case reviews suggest that longterm remission-free survival is attainable with continued usage of pexidartinib. However, the appropriate long-term dosing schedule to avoid treatment-limiting toxicity has not yet been developed. Utilization of pulsed dosing, treatment holidays, reduced dose, and the individualization of treatment schedules are all ongoing considerations.

Though significant, the risks of surgical intervention should be weighed against the expected sequelae of natural disease progression. However, the variable course of TGCT and the lack of good data on its natural history make formulating accurate predictions difficult. As discussed above, placebo patients in the ENLIVEN study did not achieve any overall tumor response and had a slight decline in their functional outcome scores. ${ }^{12}$ According to the recent review by Bernthal et al, however, the patients assigned a "monitoring" approach had lower baseline levels of stiffness and pain severity and interference than patients assigned to a surgical or medical intervention, and thus may have experienced a more rapidly progressive natural disease course. ${ }^{7}$ The relative morbidity of a treatment plan should take into account the patient's quality of life at baseline and predicted pace of disease progression. Goals of treatment are long-term eradication or control of tumor burden and improvement of musculoskeletal symptoms. Of course, the impacts considered acceptable will ultimately depend on each individual's extent of disease, age, health, and lifestyle.

Efficacious TGCT management begins with accurate diagnosis. In cases of uncertainty, such as localized disease or the presence of multiple diseases around a joint (eg, in the anterior and posterior knee compartments), or in the absence of a blooming artefact from MRI sequences (eg, susceptibility-weighted imaging or gradient echo and low B-value diffusion weighted imaging) a biopsy is needed to confirm diagnosis. After establishing a secure diagnosis, the severity of symptoms and functional limitations, and time course of the disease, need to be considered. Watchful monitoring may be pursued in some patients who have minimal symptoms and lack inflammatory joint changes. Otherwise, surgical excision should be pursued in cases in which a total or near total resection is possible. Systemic treatment (such as pexidartinib) should be employed if surgical goals are not achievable with an acceptable level of morbidity, and if the patient's medical condition allows. After 3-6 months of systemic therapy, the patient should be re-evaluated for surgical candidacy; if the disease is progressing or symptoms are not improving, reconsideration of surgery is warranted. However, if recovery markers such as pain, swelling, stiffness, and range of motion are improving, systemic treatment should be continued as long as the disease remains unresectable The combined use of neoadjuvant pexidartinib therapy and surgical resection can be implemented in the algorithm above in an attempt to reduce the likelihood of recurrence.

\section{Investigations Beyond TGCT}

In addition to its utility in TGCT, pexidartinib has the potential for applications in the treatment of other diseases. It is important to note that these developments represent preclinical work that requires further elaboration prior to clinical application.

\section{Rheumatoid Arthritis}

Pexidartinib's role in selective CSF1 inhibition has promising applications in the treatment of rheumatoid arthritis. CSF1 inhibition may dampen lipopolysaccharideinduced neuroinflammatory and bone-loss processes implicated in rheumatoid arthritis. ${ }^{42}$ Moreover, downregulation of CSF1R has been associated with inhibition of osteoclastogenesis - a process underlying bone erosion in rheumatoid arthritis - by RNA-binding protein QK15. ${ }^{43}$ Wang et al showed that in a male rat model, pexidartinib rescues lipopolysaccharide-induced destruction of bone 
microstructure, inhibits osteoclast formation, and attenuates lipopolysaccharide-induced expression of genes involved in osteoclast differentiation, including Traf6, Fral, c-fos and NFATc1. ${ }^{42}$

\section{Osteosarcoma}

CSF1 plays a crucial role in maintaining the tumorigenicity of tumor-associated macrophages by promoting macrophage proliferation and survival. ${ }^{44}$ Several studies have explored whether pexidartinib's inhibition of CSF1 could be harnessed to stall tumor progression in osteosarcoma. Studies using in vitro and patient-derived xenograft murine models with established CSF1R expression have demonstrated decreased osteosarcoma tumor growth and metastasis after treatment with pexidartinib, specifically due to decreases in CSF1R activation, ERK signaling, cell viability, and colony formation. ${ }^{45}$ Moreover, a preclinical study by Fujiwara et al found that pexidartinib reduced tumor growth and metastasis and increased metastasis-free survival in orthotropic xenograft osteosarcoma models. ${ }^{44}$ Specifically, pexidartinib treatment decreased levels of tumor-associated macrophages (TAMs) and FOXP3 regulatory $\mathrm{T}$ cells while increasing $\mathrm{CD} 8+\mathrm{T}$ cell infiltration into both primary and metastatic sites. ${ }^{44}$ It has also been shown that checkpoint immunotherapy treatment via CSF1R blockade results in promotion of CD4 and CD8 activity. ${ }^{44}$ The validation of pexidartinib's immunomodulatory effects in conjunction with previous identification of pexidartinib's action on tumor cells themselves suggest strong immunotherapeutic potential.

\section{Other Cancers}

Both preclinical and clinical studies have investigated the utility of pexidartinib as systemic and local therapy (both with or without other chemotherapy) in the context of other malignancies. Several of these efforts have focused on capitalizing on pexidartinib's interaction with TAMs. A preclinical investigation found that intrapulmonary instillation of PLX 3397 (pexidartinib) in mice with pulmonary metastases of 4T1 syngeneic breast cancers decreased CSF1R phosphorylation, increased the M1/M2 macrophage ratio, and resulted in significant tumor regression. ${ }^{46}$ Similarly, in a murine lung cancer model, local administration of pexidartinib induced a favorable shift in the M1/ M2 TAM ratio towards the M1 phenotype, which was associated with a significant reduction in tumor burden. ${ }^{47}$ The study also found that pexidartinib given in conjunction with systemic cisplatin resulted in a more robust tumor response than did either treatment alone, both in vivo and ex vivo. Pexidartinib has also been established as a potential nanoparticle drug conjugate targeting M2 macrophages in B16F10 melanoma tumors induced in mice. ${ }^{48}$ This work suggests that conjugation of pexidartinib to M2pep-coated nanoparticles can facilitate TAM uptake and bolster its anti-tumorigenic effects relative to the administration of free drug.

Pexidartinib has further been explored, in conjunction with the mTOR inhibitor sirolimus, as a means of modulating TAM polarization in unresectable soft tissue sarcoma and peripheral nerve sheath tumors. ${ }^{49}$ The preclinical precursor to this study found that malignant peripheral nerve sheath tumor (MPNST) cell lines had elevated levels of CSF1R expression and that pexidartinib reduced tumor growth in an MPNST xenograft model. Pexidartinib administered in conjunction with sirolimus led to a significant reduction in TAMs compared to pexidartinib alone. In this 24-patient, phase I study, stable disease was seen in 6 out of 18 evaluable patients treated with the combination of pexidartinib and sirolimus.

\section{Conclusions}

Pexidartinib is the first effective targeted medical therapy to provide a systemic treatment option for advanced TGCT, and is a potentially valuable alternative to surgery when the surgical approach is likely to lead to excessive morbidity. ${ }^{12}$ As the risks associated with this medication are well-documented, the optimal duration of use is unknown, and the course of treatment is often prolonged, it is crucial to identify the patients who will derive maximal benefit from its use and for whom the risks of adverse events are appropriately balanced by the avoidance of the alternative risks of surgery or observation. These predictions should be based on a patient's individual risk for severe postoperative morbidity, severity of disease at baseline, and comorbid conditions. As suggested by the TOPP study, patient-specific factors and characteristics at baseline should be strong considerations in determining treatment modality.

While the watchful monitoring approach may be appropriate for mild presentations of disease, our review of the literature confirmed that the majority of patients with symptomatic TGCT stand to benefit from surgery or a combined surgical/systemic approach. Specifically, the ENLIVEN study found that pexidartinib was efficacious in over half of patients who had undergone surgery previously and continued to experience severely symptomatic 
TGCT. ${ }^{12,33}$ Given the considerable health, economic, and social impacts of TGCT, a multifaceted approach to the disease entity is often warranted. Major contraindications to this therapy are susceptibility to hepatic failure, reproductive concerns, and concurrent use of CYP3A inhibitors and acid reducing agents. The adverse reactions associated with pexidartinib are commonly transient and reversible; furthermore, the risk profile often compares favorably with that of radiotherapy or surgery. Thus, pexidartinib represents a promising treatment to limit recurrence and improve quality of life in patients who are struggling with TGCT and for whom a surgical approach is either contraindicated or presents a greater morbidity risk than the disease itself, and to simultaneously avoid the complications associated with surgery and radiotherapy. Analysis of therapy risk and response across a broader range of demographic factors, baseline disease presentations, and comorbidity profiles, as well as a more accurate understanding of and ability to prognosticate the natural course of this disease, are needed to better evaluate the indications for pexidartinib in TGCT.

\section{Funding}

This research was funded in part through the NIH/NCI Cancer Center Support Grant P30 CA008748.

\section{Disclosure}

Anna Vaynrub and Max Vaynrub report no conflicts of interest in this work. John Healey has served as a consultant for Daiichi Sankyo and Stryker Consultant, as a trustee for the Musculoskeletal Transplant Foundation, and as a symposium editor for Clinical Orthopaedics and Related Research.

William Tap reports personal fees from Eli Lilly, EMD Serono, Mundipharma, C4 Therapeutics, Daiichi Sankyo, Blueprint, Agios Pharmaceuticals, NanoCarrier, Deciphera, Adcendo, Ayala Pharmaceuticals, Kowa, Servier, Bayer Pharmaceuticals, Epizyme, Cogent, and Medpacto outside the current article. In addition, Dr. Tap has a patent (Companion diagnostic for CDK4 inhibitors 14/854,329) pending to Memorial Sloan Kettering Cancer Center/Sloan Kettering Institute (SKCC/SKI), and a patent (Enigma and CDH18 as companion diagnostics for CDK4 inhibition - SKI2016-021-03) pending to MSKCC/SKI; is a member of the scientific advisory boards of Certis Oncology Solutions and Innova Therapeutics, and owns stock in Atropos Therapeutics and Innova Therapeutics.

\section{References}

1. Giustini N, Bernthal NM, Bukata SV, Singh AS. Tenosynovial giant cell tumor: case report of a patient effectively treated with pexidartinib (PLX3397) and review of the literature. Clin Sarcoma Res. 2018;8:14. doi:10.1186/s13569-018-0101-2

2. Staals EL, Ferrari S, Donati DM, Palmerini E. Diffuse-type tenosynovial giant cell tumour: current treatment concepts and future perspectives. Eur J Cancer. 2016;63:34-40. doi:10.1016/j.ejca.2016.04.022

3. Palmerini E, Longhi A, Donati DM, Staals EL. Pexidartinib for the treatment of adult patients with symptomatic tenosynovial giant cell tumor: safety and efficacy. Expert Rev Anticancer Ther. 2020;20 (6):441-445. doi:10.1080/14737140.2020.1757441

4. Healey JH, Bernthal NM, van de Sande M. Management of tenosynovial giant cell tumor: a neoplastic and inflammatory disease. $J \mathrm{Am}$ Acad Orthop Surg Glob Res Rev. 2020;4(11):e20.00028. doi:10.5435/ JAAOSGlobal-D-20-00028

5. Baldi GG, Gronchi A, Stacchiotti S. Pexidartinib for the treatment of adult symptomatic patients with tenosynovial giant cell tumors. Expert Rev Clin Pharmacol. 2020;13(6):571-576. doi:10.1080/ 17512433.2020.1771179

6. de Saint Aubain Somerhausen N, van de Rijn M. Synovial giant cell tumours. In: Antonescu CRW, editor. WHO Classification of Tumours: Soft Tissue and Bone Tumours. 5th ed. International Agency for Research on Cancer; 2020:133-136.

7. Bernthal NM, Spierenburg G, Healey JH, et al. The diffuse-type tenosynovial giant cell tumor (dt-TGCT) patient journey: a prospective multicenter study. Orphanet J Rare Dis. 2021;16(1):191. doi:10.1186/s13023-021-01820-6

8. Gelderblom H, Wagner AJ, Tap WD, et al. Long-term outcomes of pexidartinib in tenosynovial giant cell tumors. Cancer. 2021;127 (6):884-893. doi:10.1002/cncr.33312

9. West RB, Rubin BP, Miller MA, et al. A landscape effect in tenosynovial giant-cell tumor from activation of CSF1 expression by a translocation in a minority of tumor cells. Proc Natl Acad Sci U S A. 2006;103(3):690-695. doi:10.1073/pnas.0507321103

10. Vougiouklakis T, Shen G, Feng X, Hoda ST, Jour G. Molecular profiling of atypical tenosynovial giant cell tumors reveals novel non-CSF1 fusions. Cancers. 2019;12(1):100. doi:10.3390/cancers 12010100

11. Benner B, Good L, Quiroga D, et al. Pexidartinib, a novel small molecule CSF-1R Inhibitor in use for tenosynovial giant cell tumor: a systematic review of pre-clinical and clinical development. Drug Des Devel Ther. 2020;14:1693-1704. doi:10.2147/dddt.S253232

12. Tap WD, Gelderblom H, Palmerini E, et al. Pexidartinib versus placebo for advanced tenosynovial giant cell tumour (ENLIVEN): a randomised phase 3 trial. Lancet. 2019;394(10197):478-487. doi:10.1016/s0140-6736(19)30764-0

13. Mastboom MJL, Palmerini E, Verspoor FGM, et al. Surgical outcomes of patients with diffuse-type tenosynovial giant-cell tumours: an international, retrospective, cohort study. Lancet Oncol. 2019;20 (6):877-886. doi:10.1016/s1470-2045(19)30100-7

14. Griffin AM, Ferguson PC, Catton CN, et al. Long-term outcome of the treatment of high-risk tenosynovial giant cell tumor/pigmented villonodular synovitis with radiotherapy and surgery. Cancer. 2012;118(19):4901-4909. doi:10.1002/cncr.26529

15. Mollon B, Lee A, Busse JW, et al. The effect of surgical synovectomy and radiotherapy on the rate of recurrence of pigmented villonodular synovitis of the knee: an individual patient meta-analysis. Bone Joint J. 2015;97-b(4):550. doi:10.1302/0301-620x.97b4.34907

16. Guo Q, Shi W, Jiao C, Xie X, Jiang D, Hu Y. Results and recurrence of pigmented villonodular synovitis of the ankle: does diffuse PVNS with extra-articular extension tend to recur more often? Knee Surg Sports Traumatol Arthrosc. 2018;26(10):3118-3123. doi:10.1007/ s00167-017-4488-8 
17. Xiang X, Jiang W, Qiu C, Xiao N, Liang J. Image-guided, intensity-modulated radiotherapy for the treatment of diffuse-type tenosynovial giant cell tumor of the knee: case report and review of the literature. Medicine. 2021;100(28):e26659. doi:10.1097/ md.0000000000026659

18. Berger B, Ganswindt U, Bamberg M, Hehr T. External beam radiotherapy as postoperative treatment of diffuse pigmented villonodular synovitis. Int J Radiat Oncol Biol Phys. 2007;67(4):1130-1134. doi:10.1016/j.ijrobp.2006.10.016

19. Blanco CE, Leon HO, Guthrie TB. Combined partial arthroscopic synovectomy and radiation therapy for diffuse pigmented villonodular synovitis of the knee. Arthroscopy. 2001;17(5):527-531. doi:10.1053/jars.2001.24068

20. Horoschak M, Tran PT, Bachireddy P, et al. External beam radiation therapy enhances local control in pigmented villonodular synovitis. Int J Radiat Oncol Biol Phys. 2009;75(1):183-187. doi:10.1016/j. ijrobp.2008.10.058

21. Gortzak Y, Vitenberg M, Frenkel Rutenberg T, et al. Inconclusive benefit of adjuvant (90)yttrium hydroxyapatite to radiosynovectomy for diffuse-type tenosynovial giant-cell tumour of the knee. Bone Joint J. 2018;100-b(7):984-988. doi:10.1302/0301-620x.100b7.Bjj2017-0867.R3

22. Dürr HR, Capellen CF, Klein A, et al. The effects of radiosynoviorthesis in pigmented villonodular synovitis of the knee. Arch Orthop Trauma Surg. 2019;139(5):623-627. doi:10.1007/s00402-018-3097-4

23. Ottaviani S, Ayral X, Dougados M, Gossec L. Pigmented villonodular synovitis: a retrospective single-center study of 122 cases and review of the literature. Semin Arthritis Rheum. 2011;40(6):539-546. doi:10.1016/j.semarthrit.2010.07.005

24. Park G, Kim YS, Kim JH, et al. Low-dose external beam radiotherapy as a postoperative treatment for patients with diffuse pigmented villonodular synovitis of the knee: 4 recurrences in 23 patients followed for mean 9 years. Acta Orthop. 2012;83(3):256-260. doi:10.3109/17453674.2012.678803

25. Lin C-S, Lan K-L, Wang L-W, et al. Treatment and outcome of tenosynovial giant cell tumor/pigmented villonodular synovitis patients receiving radiotherapy in Taiwan: a single-center experience. Ther Radiol Oncol. 2020;4:14. doi:10.21037/tro-20-41

26. de Visser E, Veth RP, Pruszczynski M, Wobbes T, Van de Putte LB. Diffuse and localized pigmented villonodular synovitis: evaluation of treatment of 38 patients. Arch Orthop Trauma Surg. 1999;119(78):401-404. doi:10.1007/s004020050009

27. Cassier PA, Italiano A, Gomez-Roca CA, et al. CSF1R inhibition with emactuzumab in locally advanced diffuse-type tenosynovial giant cell tumours of the soft tissue: a dose-escalation and doseexpansion phase 1 study. Lancet Oncol. 2015;16(8):949-956. doi:10.1016/s1470-2045(15)00132-1

28. Bernthal NM, Ishmael CR, Burke ZDC. Management of pigmented villonodular synovitis (PVNS): an orthopedic surgeon's perspective. Curr Oncol Rep. 2020;22(6):63. doi:10.1007/s11912-020-00926-7

29. Gelderblom H, Cropet C, Chevreau C, et al. Nilotinib in locally advanced pigmented villonodular synovitis: a multicentre, openlabel, single-arm, phase 2 trial. Lancet Oncol. 2018;19(5):639-648. doi:10.1016/s1470-2045(18)30143-8

30. Cassier PA, Gelderblom H, Stacchiotti S, et al. Efficacy of imatinib mesylate for the treatment of locally advanced and/or metastatic tenosynovial giant cell tumor/pigmented villonodular synovitis. Cancer. 2012;118(6):1649-1655. doi:10.1002/cncr.26409

31. Smith BD, Kaufman MD, Wise SC, et al. Vimseltinib: a precision CSF1R therapy for tenosynovial giant cell tumors and diseases promoted by macrophages. Mol Cancer Ther. 2021;20(11):2098-2109. doi:10.1158/1535-7163.Mct-21-0361

32. Lewis JH, Gelderblom H, van de Sande M, et al. Pexidartinib long-term hepatic safety profile in patients with tenosynovial giant cell tumors. Oncologist. 2021;26(5):e863-e873. doi:10.10 02/onco.13629
33. Tap WD, Wainberg ZA, Anthony SP, et al. Structure-guided blockade of CSF1R kinase in tenosynovial giant-cell tumor. $N$ Engl J Med. 2015;373(5):428-437. doi:10.1056/NEJMoa1411366

34. Van De Sande M, Tap WD, Gelhorn HL, et al. Pexidartinib improves physical functioning and stiffness in patients with tenosynovial giant cell tumor: results from the ENLIVEN randomized clinical trial. Acta Orthop. 2021;92(4):493-499. doi:10.1080/17453674.2021.1922161

35. Speck RM, Ye X, Bernthal NM, Gelhorn HL. Psychometric properties of a custom patient-reported outcomes measurement information system (PROMIS) physical function short form and worst stiffness numeric rating scale in tenosynovial giant cell tumors. J Patient Rep Outcomes. 2020;4(1):61. doi:10.1186/s41687-020-00217-6

36. Turalio. Prescribing Information. Daiichi Sankyo, Inc; 2019.

37. European Medicines Agency. Turalio. Available from: https://www.ema. europa.eu/en/medicines/human/EPAR/turalio. Accessed September 15, 2021.

38. Smith CC, Levis MJ, Frankfurt O, et al. A phase $1 / 2$ study of the oral FLT3 inhibitor pexidartinib in relapsed/refractory FLT3-ITD-mutant acute myeloid leukemia. Blood Adv. 2020;4(8):1711-1721. doi:10.1182/bloodadvances.2020001449

39. Lee JH, Chen TW, Hsu CH, et al. A phase I study of pexidartinib, a colony-stimulating factor 1 receptor inhibitor, in Asian patients with advanced solid tumors. Invest New Drugs. 2020;38(1):99-110. doi:10.1007/s10637-019-00745-z

40. US Food and Drug Administration. Approved risk evaluation and mitigation strategies (REMS). Available from: https://www.access data.fda.gov/scripts/cder/rems/index.cfm?event=IndvRemsDetails. page\&REMS=389. Accessed September 15, 2021.

41. Monestime S, Lazaridis D. Pexidartinib (TURALIO ${ }^{\mathrm{TM}}$ ): the first FDA-indicated systemic treatment for tenosynovial giant cell tumor. Drugs R D. 2020;20(3):189-195. doi:10.1007/s40268-020-00314-3

42. Wang XF, Wang YJ, Li TY, et al. Colony-stimulating factor 1 receptor inhibition prevents against lipopolysaccharide -induced osteoporosis by inhibiting osteoclast formation. Biomed Pharmacother. 2019;115:108916. doi:10.1016/j.biopha.2019.108916

43. Denny WA, Flanagan JU. Small-molecule CSF1R kinase inhibitors; review of patents 2015-present. Expert Opin Ther Pat. 2021;31 (2):107-117. doi:10.1080/13543776.2021.1839414

44. Fujiwara T, Yakoub MA, Chandler A, et al. CSF1/CSF1R signaling inhibitor pexidartinib (PLX3397) reprograms tumor-associated macrophages and stimulates T-cell infiltration in the sarcoma microenvironment. Mol Cancer Ther. 2021;20(8):1388-1399. doi:10.1158/ 1535-7163.Mct-20-0591

45. Smeester BA, Slipek NJ, Pomeroy EJ, et al. PLX3397 treatment inhibits constitutive CSF1R-induced oncogenic ERK signaling, reduces tumor growth, and metastatic burden in osteosarcoma. Bone. 2020;136:115353. doi:10.1016/j.bone.2020.115353

46. Alhudaithi SS, Almuqbil RM, Zhang H, et al. Local targeting of lungtumor-associated macrophages with pulmonary delivery of a CSF-1R inhibitor for the treatment of breast cancer lung metastases. Mol Pharm. 2020;17(12):4691-4703. doi:10.1021/acs.molpharmaceut. 0c00983

47. Zhang H, Almuqbil RM, Alhudaithi SS, Sunbul FS, da Rocha SRP. Pulmonary administration of a CSF-1R inhibitor alters the balance of tumor-associated macrophages and supports first-line chemotherapy in a lung cancer model. Int J Pharm. 2021;598:120350. doi:10.1016/ j.ijpharm.2021.120350

48. Pang L, Pei Y, Uzunalli G, Hyun H, Lyle LT, Yeo Y. Surface modification of polymeric nanoparticles with M2pep peptide for drug delivery to tumor-associated macrophages. Pharm Res. 2019;36(4):65. doi:10.1007/s11095-019-2596-5

49. Manji GA, Van Tine BA, Lee SM, et al. A phase I study of the combination of pexidartinib and sirolimus to target tumor-associated macrophages in unresectable sarcoma and malignant peripheral nerve sheath tumors. Clin Cancer Res. 2021;27(20):5519-5527. doi:10.11 58/1078-0432.Ccr-21-1779 


\section{Publish your work in this journal}

OncoTargets and Therapy is an international, peer-reviewed, open access journal focusing on the pathological basis of all cancers, potential targets for therapy and treatment protocols employed to improve the management of cancer patients. The journal also focuses on the impact of management programs and new therapeutic

Submit your manuscript here: https://www.dovepress.com/oncotargets-and-therapy-journal agents and protocols on patient perspectives such as quality of life, adherence and satisfaction. The manuscript management system is completely online and includes a very quick and fair peer-review system, which is all easy to use. Visit http://www.dovepress.com/ testimonials.php to read real quotes from published authors. 